\title{
A Multiobjective Game Approach with a Preferred Target Based on a Leader-Follower Decision Pattern
}

\author{
Neng-gang Xie $\mathbb{D}^{1},{ }^{1}$ Zhong Chen, ${ }^{2}$ Kang Hao Cheong, ${ }^{3}$ Rui Meng, and Wei Bao ${ }^{1}$ \\ ${ }^{1}$ School of Mechanical Engineering, Anhui University of Technology, Ma'anshan, Anhui Province, China \\ ${ }^{2}$ Zhongtian Construction Group Co., Hangzhou, Zhejiang Province, China \\ ${ }^{3}$ Engineering Cluster, Singapore Institute of Technology, 10 Dover Drive, Singapore 138683 \\ Correspondence should be addressed to Neng-gang Xie; xienenggang@aliyun.com
}

Received 5 September 2017; Revised 26 January 2018; Accepted 7 February 2018; Published 20 March 2018

Academic Editor: Vladimir Turetsky

Copyright (C) 2018 Neng-gang Xie et al. This is an open access article distributed under the Creative Commons Attribution License, which permits unrestricted use, distribution, and reproduction in any medium, provided the original work is properly cited.

We propose a multiobjective leader-follower game based on the Stackelberg model, where the designer's preferred target is taken into account. Here, the preferred target is regarded as a leader and the other targets are regarded as followers. A partition method of strategy subspace is also given. Finally, a real-life example of the multiobjective optimization design of a Chinese arch dam named "Baihetan" is presented to demonstrate the effectiveness of our proposed method.

\section{Introduction}

Given the similarity between the multiobjective design and game theory, game theory has been applied to solve many multiobjective design problems in recent years. The crux of the multiobjective method is to establish the mapping relationships between the elements of the multiobjective optimization model and the elements of the game. The multiobjective optimization model includes design objectives, objective functions, design variables, and constraints, while the game elements contain game players, benefit functions, strategy subspaces, and constraints. Two mapping relationships are listed as follows.

(1) The Space Mapping. Design variable space $\mathbf{X}=\left\{x_{1}, x_{2}, \ldots\right.$, $\left.x_{n}\right\}$ is divided into the parallel strategy subspaces $S_{1}=\left\{x_{i}, \ldots\right.$, $\left.x_{j}\right\}, \ldots, S_{m}=\left\{x_{k}, \ldots, x_{l}\right\}$ owned by each game player. It satisfies $S_{1} \cup \cdots \cup S_{m}=X ; S_{a} \cap S_{b}=0(a, b=1, \ldots, m ; a \neq b)$.

(2) The Bionic Mapping. There are $m$ design objectives, which are modeled as $m$ game players with a certain intelligence. The design objectives are given the behaviors of the game players (such as competition, cooperation, and adaptive behaviors). The interdependent game pattern of each design goal is formed, and the quantitative mapping relationships between the benefit functions of the game and the objective functions are constructed according to the qualitative description characteristics of different behavioral patterns. The constraints of the multiobjective optimization model also correspond to the constraints of the model of the game.

The conventional multiobjective optimization method usually adopts the method of "combination" for the objective function, while the game approach uses "partition" to deal with the problem of the design variables. At present, the space partition approaches of design variables include the fuzzy clustering method proposed by Wang et al. [1], a sensitivity analysis method proposed by $\mathrm{Hu}$ and Rao [2], and a step adjustment method by Clarich et al. [3].

It is well known that the key issue of the multiobjective optimization model is to take into account the interests of each target and to reflect their status. For the multiobjective game, the particular embodiment is to give each design objective the appropriate behavior patterns and construct game patterns between design objectives. Regarding the behavior patterns of the design objectives, there are mainly two types, one is the "egoistic" type with the competitive behavior, and the other is the "you have me, I have you" type of cooperative behavior. Furthermore, there exist four kinds of game patterns (pure competitive game pattern [4-9], pure cooperative game pattern [9-11], hybrid game pattern [3], and evolution game pattern [12]). In the pure competitive 
game pattern, all the game players (design objectives) obtain game profits through a competitive behavior. In the pure cooperative game pattern, all the game players gain game profits through a cooperative behavior. In the hybrid game pattern, some game players achieve game profits through a competitive behavior, but some game players obtain game profits through a cooperative behavior. In the evolutionary game pattern, the behavior of all game players is automatically adjusted based on evolution rules.

In engineering practice, there exists a kind of multiobjective problems where the designers have a preferred target, and their target status may be unequal. There are many processing methods based on the conventional multiobjective optimization, for instance, the weighted sum method by adjusting the target weights to reflect the preferred target and the hierarchical sequence method by adjusting the target optimization order according to the target's preference. Using game theory to solve the multiobjective optimization design problems with the preference for the target, Wang et al. [13] proposed Stackelberg genetic algorithms based on the Stackelberg equilibrium to solve problems in Aerospace Engineering involving high lift multiairfoil systems. In this paper, we present a method of a "leader-follower" game pattern for a target preference according to Stackelberg model. In Section 2, we will discuss the theories related to the Stackelberg model. In Section 3, a simple numerical example will be presented. The results will be compared with those found in [13]. In Section 4, a real-life example of the multiobjective optimization design of an arch dam is given.

\section{A Multiobjective Method Based on a Leader-Follower Game Pattern}

2.1. A Leader-Follower Decision and a Stackelberg Model. For a leader-follower decision problem, each decision-maker is in a different level. Each decision-maker has its own objective function. The higher level decision-maker is endowed with a more important objective function, so the final decision is often a coordinated result which the decision makers at all levels seek. Under this scheme, the goal of the top decisionmaker can be optimized, and the goal of the lower level decision-maker can be optimized in the subordinate position.

The decision making problem with a leader-follower hierarchical structure was first proposed by a German economist V. Stackelberg in 1952 when studying market economy problems. Therefore, the leader-follower decision problem is also called Stackelberg game. A typical application of the Stackelberg model exists in the oligopoly market in economics. We assume two producers in the oligopoly market where one producer is a leader, and the other one is the follower. The game aims to minimize their cost function. The leader gives priority to the decision. The follower must make its own decision following the decision made by the leader.
We define $F_{S}$ as the game profit (cost function) of the leader and $F_{W}$ as the game profit of the follower. $S_{S}$ is defined as the strategy space of the leader and $S_{W}$ as the strategy space of the follower. Let $s_{S} \in S_{S}$ and $s_{W} \in S_{W}$ be an arbitrary strategy. If there exists $\sup _{s_{W} \in R\left(s_{S}^{*}\right)} F_{S}\left(s_{S}^{*}, s_{W}\right) \leq$ $\sup _{s_{W} \in R\left(s_{S}\right)} F_{S}\left(s_{S}, s_{W}\right) \forall s_{S} \in S_{S}$, then $s_{S}^{*} \in S_{S}$ is called the Stackelberg strategy of the leader.

The leader can achieve its lower bound $F_{S}^{*}=$ $\inf _{s_{S} \in S_{S}}\left\{\sup _{s_{W} \in R\left(s_{S}\right)} F_{S}\left(s_{S}, s_{W}\right)\right\}$, where $R\left(s_{S}\right)=\left\{s_{W}^{*} \mid F_{W}\left(s_{S}\right.\right.$, $\left.\left.s_{W}^{*}\right) \leq F_{W}\left(s_{S}, s_{W}\right), \forall s_{W} \in S_{W}\right\}$ is the reaction function of the follower in relation to the leader.

The presence of leader and follower in the Stackelberg oligopoly game means that the satisfaction of the game players will be different. The leader can obtain a higher satisfaction level than the follower. Hence, for the multiobjective optimization with a preferred target, the preferred target is regarded as the leader, and the other targets are taken as the follower. If there are $m(m>2)$ oligopoly markets, then $m-1$ followers are in the subordinate position. For a reallife example of the multiobjective optimization design of an arch dam, we have three objectives where one preferred target will be the leader with the other two targets as the subordinate position. The corresponding game profit of the followers $F_{W}$ is a weighted combination of these two objectives.

The decision mechanism of the Stackelberg model is as follows: the leader first announces the strategy of making its objective function optimal, which will influence the constraint set and the objective function of the follower's optimal decision. Then, the follower selects the strategy $s_{W}$ to make its objective function reach the optimum under this premise. Since the choice of the follower affects the constraint and the objective function of the leader's optimal decision. The leader can further adjust its strategic variables $s_{S}$. The process will repeat until the leader's objective function, $F_{S}$, is optimal.

\subsection{Solution Steps}

2.2.1. Exploration Method of the Strategy Space. A spatial game approach has been proposed in [14], which can sort the items and provide a way to simplify the knapsack problem. For the multiobjective optimization problem based on the game approach, design variables are first required to be divided into multiple strategy subspaces owned by game players. Here, we establish related concepts from [14] (such as the space distance and sorting methods) to propose a novel exploration method involving game player's strategy space [15]. The computation steps are as follows.

Algorithm 1. (1) Optimize $m$ single objective, and then obtain optimal solution $f_{1}\left(\mathbf{X}_{1}^{*}\right), f_{2}\left(\mathbf{X}_{2}^{*}\right), \ldots, f_{m}\left(\mathbf{X}_{m}^{*}\right)$, where $\mathbf{X}_{i}^{*}=$ $\left\{x_{1 i}^{*}, x_{2 i}^{*}, \ldots, x_{n i}^{*}\right\}(i=1,2, \ldots, m)$.

(2) Every $x_{j}$ is divided into $T$ fragments with a step length $\Delta x_{j}$ in its feasible space. The effect of $x_{j}$ on the objective $f_{i}$ is first computed as follows:

$$
\Theta(j, i)=\frac{\sum_{t=1}^{T}\left|f_{i}\left(x_{1 i}^{*}, \ldots, x_{(j-1) i}^{*}, x_{j}(t), x_{(j+1) i}^{*}, \ldots, x_{n i}^{*}\right)-f_{i}\left(x_{1 i}^{*}, \ldots, x_{(j-1) i}^{*}, x_{j}(t-1), x_{(j+1) i}^{*}, \ldots, x_{n i}^{*}\right)\right|}{T \cdot \Delta x_{j}} .
$$


The normalization gives an impact index $\Delta(j, i)$, which is defined below:

$$
\begin{aligned}
\Delta(j, i)=\frac{\Theta(j, i)}{\sum_{l=1}^{n} \Theta(l, i)} & \\
& (j=1,2, \ldots, n ; i=1,2, \ldots, m) .
\end{aligned}
$$
follows:

(3) $d(j, i)$ is defined as the space distance from $x_{j}$ to $f_{i}$ as

$$
\begin{aligned}
d(j, i)=\frac{1 / \Delta(j, i)}{\sum_{h=1}^{m}(1 / \Delta(j, h))} & \\
& (j=1,2, \ldots, n ; i=1,2, \ldots, m) .
\end{aligned}
$$

Also, $\operatorname{Mo}(j)$ is defined as the moment of $x_{j}$ to all objective functions, which represents the full influence degree of $x_{j}$ on all objective functions:

$$
\operatorname{Mo}(j)=\frac{1}{\sum_{h=1}^{m}(1 / \Delta(j, h))} \quad(j=1,2, \ldots, n) .
$$

$\lambda$ is defined as the threshold of moment:

$$
\lambda=\frac{\sum_{j=1}^{n} \operatorname{Mo}(j)}{2} \text {. }
$$

(4) All design variables assigned to each objective function (each game player) are sorted according to the descending order of $d(j, i)$.

(5) Each game player first chooses a design variable being the first ranking and then the second ranking and so on until the accumulative moment of the selected variables is greater than or equal to the threshold of moment, $\lambda$.

As an illustration, the partition rules of design variables are performed as follows:

(a) If one chosen design variable $x_{j}(j=1,2, \ldots, n)$ has a ranking different from the game players, then this design variable is assigned to one game player with a relatively higher ranking;

(b) If one design variable $x_{j}(j=1,2, \ldots, n)$ has the same highest ranking among the multiple game players, the ownership of this design variable is determined by the impact index. In particular, if $\Delta(j, i)$ is the greatest, then $x_{j}$ is assigned to $f_{i}$.

(c) According to the partition rules, all the design variables are assigned to the corresponding game players (objective functions).

2.2.2. Game Algorithm. According to the above decision mechanism based on the Stackelberg model, the steps of the multiobjective game based on the leader-follower game pattern can be described as follows.

Algorithm 2. (1) Obtain the strategy space $S_{1}, \ldots, S_{m}$ attached to each player according to the method in Section 2.2.1 and then form $S_{S}$ (the strategy space of the leader) and $S_{W}$ (the strategy space of the follower).

(2) Generate $L$ sets of the initial feasible strategies $s_{i}^{(0)}=$ $\left\{s_{S i}^{(0)}, s_{W i}^{(0)}\right\}(i=1, \ldots, L)$. Here, $s_{S i}^{(0)}(i=1, \ldots, L)$ is the initial feasible strategy of the leader and $s_{W i}^{(0)}(i=1, \ldots, L)$ is the initial feasible strategy of the follower.

(3) For any $s_{S i}^{(0)}(i=1, \ldots, L)$, optimize $F_{W}$ in $S_{W}$, and obtain $s_{W i}^{R}(i=1, \ldots, L)$, which is the "follower strategy" of the follower obeying the leader.

(4) For any $s_{W i}^{R}(i=1, \ldots, L)$, optimize $F_{S}$ in $S_{S}$, and obtain $s_{S i}^{R}(i=1, \ldots, L)$, which is the "response strategy" of the leader to the follower.

(5) Define the strategy combination $s_{i}^{(1)}=s_{S i}^{R} \cup s_{W i}^{R}$ and construct the fitness function $\left\|S_{S i}^{R}-S_{S i}^{(0)}\right\| . s_{\min }^{(1)}$ is the strategy combination with a minimum fitness function value.

(6) If $\left|\left(F_{S}\left(s_{\text {min }}^{(k)}\right)-F_{S}\left(s_{\text {min }}^{(k-1)}\right)\right) / F_{S}\left(s_{\text {min }}^{(k)}\right)\right| \leq \varepsilon(k=1,2, \ldots)$ ( $k$ refers to the number of iterations; $\varepsilon$ is a decimal parameter given in advance; when $\left.k=0, F_{S}\left(s_{\text {min }}^{(0)}\right)=\min _{i=1,2, \ldots, L} F_{S}\left(s_{i}^{(0)}\right)\right)$, then a solution is produced, and the algorithm terminates. If it is not satisfied, then the leader's strategy of the $k_{\text {th }}$ generation $s_{S i}^{(k)}(i=1,2, \ldots, L)$ is generated according to (6) given below. Return to step (3) of Algorithm 2 to perform a loop.

$$
\begin{array}{r}
\rho_{i j}^{(k)}=\rho_{i j}^{(k-1)} \exp \left(\theta^{\prime} \cdot N(0,1)+\theta \cdot N_{j}(0,1)\right) \\
(j=1,2, \ldots, H ; i=1,2, \ldots, L) \\
x_{i j}^{(k)}=x_{i j}^{(k-1)}+N\left(0, \rho_{i j}^{(k)}\right) \\
(j=1,2, \ldots, H ; i=1,2, \ldots, L),
\end{array}
$$

where $x_{i j}^{(k)}$ and $x_{i j}^{(k-1)}$ denote the $j_{\text {th }}$ component of the $i_{\text {th }}$ strategy of the leader in a $k_{\text {th }}$ and $(k-1)_{\text {th }}$ generation, respectively. $H$ represents the number of design variables owned by the leader. $\rho_{i j}^{(k)}$ and $\rho_{i j}^{(k-1)}$ are referred to as the variance variable and $\rho_{i j}^{(0)}=1(j=1,2, \ldots, H ; i=1,2, \ldots, L)$, $\theta^{\prime}=1 / \sqrt{2 L}, \theta=1 / \sqrt{2 \sqrt{L}}, N(0,1)$, and $N_{j}(0,1)$ are the independent standard normal random variables. $N\left(0, \rho_{i j}^{(k)}\right)$ is also a normal random variable.

\section{Test Example}

A simple test case will be used to illustrate the process of the multiobjective optimization proposed in this paper. We have the following equations:

$$
\begin{aligned}
& \min f_{1}=(x-1)^{2}+(x-y)^{2} \\
& \min f_{2}=(y-3)^{2}+(x-y)^{2}
\end{aligned}
$$

$$
(-5 \leq x, y \leq 5) \text {. }
$$

Optimize single objective: $f_{1}^{*}=0.0 ; x_{11}^{*}=1.0 ; x_{21}^{*}=$ $1.0 ; f_{2}^{*}=0.0 ; x_{12}^{*}=3.0 ; x_{22}^{*}=3.0$. Impact index $\Delta(j, i)$, space distance $d(j, i)$, and moment $\operatorname{Mo}(j)$ are computed, and all design variables are sorted according to Section 2.2.1. The related results are shown in Table 1.

The threshold of moment is computed according to (5) and determined to be 0.222 . According to Table 1 and step (5) of Algorithm 1 in Section 2.2.1, we will then have $S_{1}=\left\{x_{1}\right\}$ and $S_{2}=\left\{x_{2}\right\}$. 
TABLE 1: Computation results of a simple test case.

\begin{tabular}{|c|c|c|c|c|c|c|c|}
\hline$x_{j}$ & $\Delta(j, 1)$ & $\begin{array}{c}f_{1} \\
d(j, 1)\end{array}$ & Ranking & $\Delta(j, 2)$ & $\begin{array}{c}f_{2} \\
d(j, 2)\end{array}$ & Ranking & $\operatorname{Mo}(j)$ \\
\hline$x_{1}$ & 0.667 & 0.333 & 1 & 0.333 & 0.667 & 2 & 0.222 \\
\hline$x_{2}$ & 0.333 & 0.667 & 2 & 0.667 & 0.333 & 1 & 0.222 \\
\hline
\end{tabular}

The numerical results can be easily obtained by following Algorithm 2 in Section 2.2.2. These results are then compared with those obtained in [13], as well as the results obtained by the Stackelberg game given in [13]. Table 2 shows that our proposed method is better than the method in [13] for this simple test (because both the leader's objective value and the follower's objective value are better than those in [13]).

\section{Case Study: A Triobjective Shape Optimization Design for an Arch Dam}

The shape of an arch dam will have a large influence on the volume, stress, and displacement of the dam. Hence, the shape of an arch dam determines the economic aspects and its safe operation. To ensure the safety and economy viability of a high arch dam to the static load case, many have carried out studies on the multiobjective optimization design of an arch dam [15-18]. These studies focus on the construction of the geometric model, selection of design variables, defining the objective functions, constraint conditions, structural analysis, and optimization method.

4.1. Model Formulation. In our model, the continuous geometry is modeled such that the shape of the horizontal arch and shape of the arch crown section of an arch dam are described separately like in [15]. Here, the shape of the horizontal arch is determined by the curves of the upper and lower planes of the arch, which can be easily defined by the equations of the arch axis and the arch thickness. The shape of the crown beam section is determined by the curves of the upper and lower planes of the arch crown beam and can be defined by the curve equations of the upstream surface of the arch crown beam and the thickness equations of the arch crown beam. According to established geometric model of the arch dam, the design variables of the shape are determined to be $\mathbf{X}=\left\{x_{1}, x_{2}, \ldots, x_{n}\right\}$.

The dam volume $V$ is defined to be the economic objective; $f_{1}(\mathbf{X})=V$. The maximum principal tensile stress (of the dam) is the safety goal of arch dam's local area, $f_{2}(\mathbf{X})=\max _{V}\left(\sigma_{1}\right)$, and $\sigma_{1}$ is the principal tensile stress. The strain energy $U$ of the arch dam is taken as the overall safety goal; $f_{3}(\mathbf{X})=U$. There are also other constraints like the geometric constraints, stress constraints, stability constraints, and volume constraints. The finite-element method was primarily used for the structure analysis.

A triobjective shape optimization model for an arch dam is given as follows:
Seeking the design variables

$$
\mathbf{X}=\left\{\begin{array}{llll}
x_{1} & x_{2} & \cdots & x_{n}
\end{array}\right\}
$$

Let objective functions be

$$
F(\mathbf{X})=\left\{V \max _{V}\left(\sigma_{1}\right) U\right\} \longrightarrow \min
$$

\section{And satisfy the following constraints:}

Volume constraints: $V_{\min } \leq V \leq V_{\max }$ (arch dam volume does not exceed the allowable value)

Geometric constraints: $\left\{T_{\min } \leq T \leq T_{\max }\right.$ (arch dam thickness $T$ does not exceed the allowable value); $K_{u} \leq\left[K_{u}\right.$ ] (upstream overhang degree $K_{u}$ does not exceed the allowable value); $K_{d} \leq$ $\left[K_{d}\right]$ (downstream overhang degree $K_{d}$ does not exceed the allowable value)\}

Stress constraints: $\min _{V} \sigma_{3} \geq\left[\sigma_{3}\right]$ (the maximum principal compressive stress $\boldsymbol{\sigma}_{3}$ does not exceed the allowable value)

Stability constraints: $\varphi_{\max } \leq\left[\varphi_{\max }\right]$ (the maximum central angle $\varphi_{\max }$ does not exceed the allowable value)

where $U$ is the strain energy, $U=(1 / 2) \boldsymbol{\delta}^{T} K \boldsymbol{\delta} . \boldsymbol{\delta}$ represents the displacement matrix of the whole nodes and $K$ represents the whole stiffness matrix.

Here, a Chinese arch dam named "Baihetan" (ready for construction) is used as a way of example to illustrate the effectiveness of our proposed method. Furthermore, a static analysis of the arch dam-water-foundation rock system is under a gravity load and hydrostatic pressure. The foundation rock is assumed to be massless [19-21]. The calculations related to the foundation rock using finite-element analysis are as follows: (1) the foundation rock in upstream and depth directions is calculated at twice the height of the dam. (2) The downstream side and the left and right sides of the foundation rock are calculated to be two times higher than the dam. The foundation rock is simulated by the linear elastic material, where the elastic modulus is $2.0 \times 10^{4} \mathrm{MPa}$ and Poisson's ratio is 0.2 . The parabolic-hyperbolic shape of the arch dam is first considered, and the elevation of dam bottom is $550.0 \mathrm{~m}$ with a height of $277.0 \mathrm{~m}$. The upstream normal storage level is $820.0 \mathrm{~m}$, the static elastic modulus is $2.10 \times$ $10^{4} \mathrm{MPa}$, Poisson's ratio is 0.167 , and its density is $2.4 \mathrm{t} / \mathrm{m}^{3}$. The finite-element method is used for the structural analysis, where a hexahedron shape with 20 nodes and a pentahedron shape with 15 nodes are used. A two-layer element is arranged along the direction of the dam thickness, and a five-layer element is arranged along the direction of the dam height.

The upstream curves of the arch dam and the thickness of the crown cantilever are simulated using cubic curves, and the other geometry characteristics are simulated using the Lagrange interpolation equation. There are 39 design variables. The design variables distribution, upper and lower limit, and the initial shape parameters are shown in Table 3, provided by a design institution. The constraints are as 
TABLE 2: Comparisons of the analytical and numerical results.

\begin{tabular}{llcccc}
\hline & & $x_{1}$ & $x_{2}$ & Objective value, $f_{1}$ & Objective value, $f_{2}$ \\
\hline \multirow{2}{*}{ Stackelberg game [13] } & Preferred $f_{1}$ & 1.4 & 2.2 & 0.8 & 1.28 \\
& Preferred $f_{2}$ & 1.8 & 2.6 & 1.28 & 0.8 \\
Method given in [13] & Preferred $f_{1}$ & 1.399945 & 2.200030 & 0.800092 & 1.280002 \\
& Preferred $f_{2}$ & 1.799998 & 2.600001 & 1.280001 & 0.800004 \\
This paper & Preferred $f_{1}$ & 1.409656 & 2.202957 & 0.797145 & 1.264605 \\
& Preferred $f_{2}$ & 1.800240 & 2.597472 & 1.275963 & 0.797608 \\
\hline
\end{tabular}

follows: (1) $V_{\min }=600 \times 10^{4} \mathrm{~m}^{3}$ and $V_{\max }=750 \times 10^{4} \mathrm{~m}^{3}$; (2) the upstream overhang degree: $K_{u} \leq 0.3$; the downstream overhang degree: $K_{d} \leq 0.25$; (3) the largest central angle: $\varphi_{\max }<100^{\circ}$; (4) the chord length and quasi semicentral angle constraints; (5) the allowable principal compressive stress value is given as $\left[\sigma_{3}\right]=-15.0 \mathrm{MPa}$.

The impact index, space distance, and space moment are computed, and all design variables are sorted according to the rules set out in Section 2.2.1. According to the partition rules, the strategy subspace of the game player $\left(f_{1}\right): S_{1}=\left\{x_{16} \sim\right.$ $\left.x_{23}, x_{32} \sim x_{39}\right\}$, the strategy subspace of the game player $\left(f_{2}\right)$ : $S_{2}=\left\{x_{8} \sim x_{15}, x_{24} \sim x_{31}\right\}$, and the strategy subspace of the game player $\left(f_{3}\right): S_{3}=\left\{x_{1} \sim x_{7}\right\}$.

When leader-follower game method is used to solve optimization problems with three objective functions, the preferred target is regarded as the leader, and the other two targets are taken as the follower. For example, when $f_{1}$ is regarded as preferred target, the game profit of the leader $F_{S}=f_{1}$ and the game profit of the follower $F_{W}=0.5\left(f_{2} / f_{2}^{*}\right)+$ $0.5\left(f_{3} / f_{3}^{*}\right)$. The strategy space of the leader is $S_{S}=S_{1}$ and the strategy space of the follower is $S_{W}=S_{2} \cup S_{3}$. The situation is handled in the same way if $f_{2}$ or $f_{3}$ is regarded as the preferred target.

4.2. Numerical Results. The main parameters of the initial shape and the shape featured in leader-follower game are shown in Table 4. There are three kinds of leader-follower game shape parameters, and their respective results are presented in Tables 5, 6, and 7. Results in the tables show the shape of the preferred $f_{1}$ is the smallest and the corresponding thickness of arch dam is minimal. On comparison, the shape of the preferred $f_{2}$ appears to be more curvy with its crown cantilever section towards the downstream. Such features have the potential to reduce the value of the maximum principal tensile stress. The shape of the preferred $f_{3}$ appears to be more upright, with its crown cantilever section towards the upstream, which can reduce the dam deformation and decrease deformation energy. In Figure 1, the upstream element strain energy is compared with various preferred targets. The downstream element strain energy is compared with various preferred targets in Figure 2. Figures 1 and 2 show that the distribution of the element deformation energy is suitably reasonable when $f_{3}$ is selected as the preferred target. Here, the deformation energy at the bottom of the dam is large due to the relatively thicker dam's bottom and the deformation energy at the top of the dam is small due to the relatively thinner dam's top. The displacement along the river of the downstream surface is compared with different preferred targets in Figure 3. The contour line of the upstream surface principal tensile stress is compared with various preferred targets in Figure 4. The comparison of the contour line of downstream area principal compressive stress with various preferred targets is shown in Figure 5. Figures 3,4 , and 5 indicate that the distributions of the displacement and the stress are identical, but the numerical size is different.

Our numerical results have demonstrated that all three shapes based on the leader-follower game can achieve their preferred targets. Compared to the initial shape (see Table 4), the shape of the preferred $f_{1}$ (volume) is reduced by $667500 \mathrm{~m}^{3}$ (about $9.68 \%$ ). The shape of the preferred $f_{2}$ (stress) is reduced by $1.56 \mathrm{MPa}$ (about $13.48 \%$ ). The shape of the preferred $f_{3}$ (energy) is reduced by $0.455 \mathrm{GJ}$ (about $12.19 \%)$. These results have highlighted the status of the preferred targets and demonstrated the effectiveness of our proposed method. Besides, the maximum center angle of the arch dam is related to the stability of the arch abutment and the dam shoulder. The smaller maximum center angle yields a better stability of the arch abutment and the dam shoulder. The shape of the preferred $f_{3}$ appears to be more robust (Table 4 shows that the maximum center angle is $95.104^{\circ}$, which is the smallest among the four shapes), and its multiobjective functions have been further improved as well.

\section{Conclusion}

(1) In this paper, we have developed an original and novel Stackelberg model in solving a multiobjective design problem with the preferred target. In our model, we utilize a method for computing the strategy subspace. A multiobjective game based on the leader-follower game pattern has also been established. Our optimization example of an arch dam with three targets shows that our approach is efficient and can implement preferred objective (among other objectives) in a leader-follower game-theoretic manner.

(2) From the process of the leader-follower game we notice that the leader player gives priority to a decision making. After the leader player makes the decision, the follower player selects its own strategy variables according to the decision of the leader player to optimize its goal. Therefore, the leader player restricts the achievement of the subordinate goal, and the decision of the follower player will also influence the goal of the leader player. The optimization 


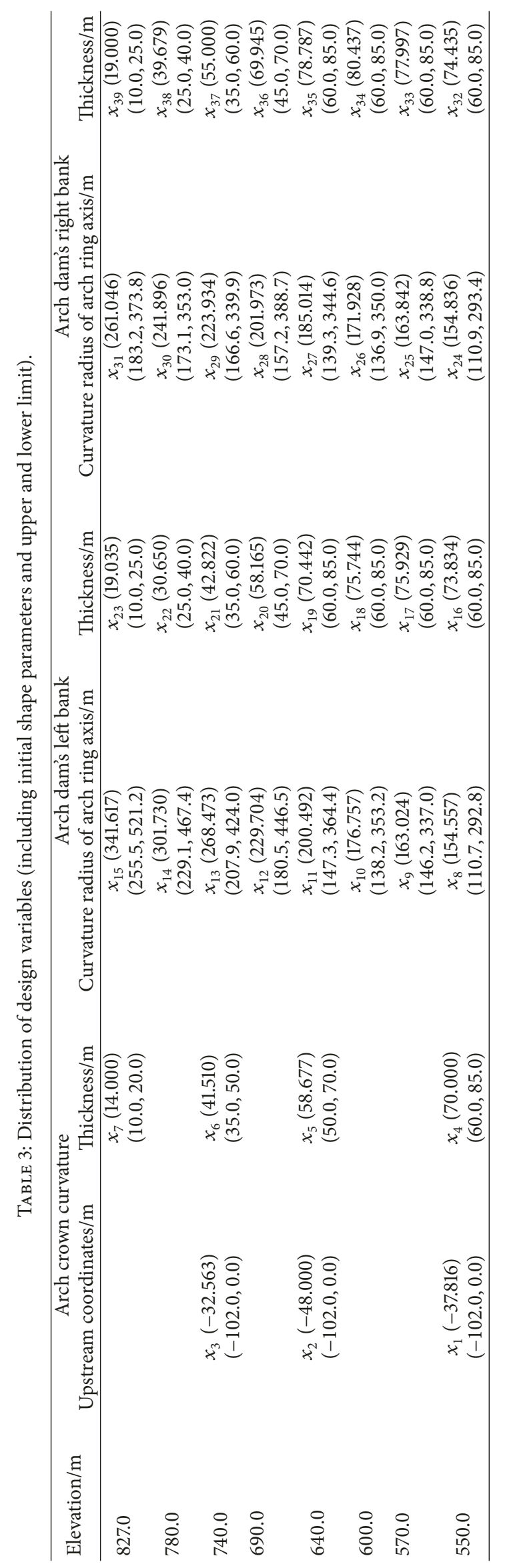


TABLE 4: The main technical parameters of the initial shape and leader-follower game shape.

\begin{tabular}{|c|c|c|c|c|c|c|c|}
\hline & & Volume $/ 10^{4} \mathrm{~m}^{3}$ & Strain energy/GJ & The maximum principal tensile stress/MPa & $K_{u}$ & $K_{d}$ & $\varphi_{\max }{ }^{\circ}$ \\
\hline \multirow{4}{*}{ Game shape } & shape & 689.26 & 3.733 & 11.57 & 0.254 & 0.048 & 96.312 \\
\hline & Preferred $f_{1}$ & 622.51 & 4.088 & 12.57 & 0.127 & 0.113 & 98.912 \\
\hline & Preferred $f_{2}$ & 723.65 & 4.273 & 10.01 & 0.213 & 0.145 & 99.754 \\
\hline & Preferred $f_{3}$ & 697.90 & 3.278 & 11.52 & 0.186 & 0.123 & 95.104 \\
\hline
\end{tabular}

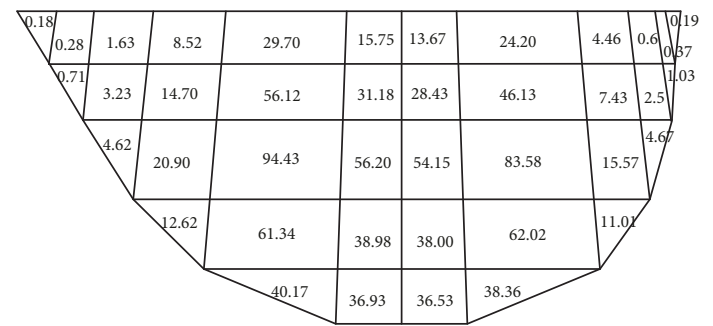

(a) Initial shape

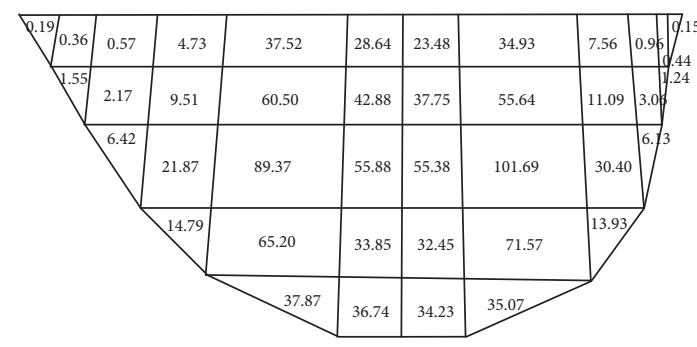

(c) Leader-follower game shape (preferred $f_{2}$ )

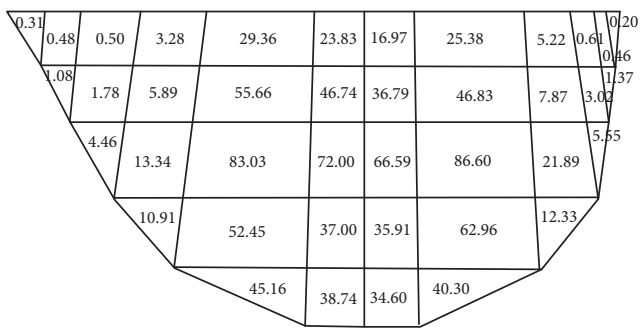

(b) Leader-follower game shape (preferred $f_{1}$ )

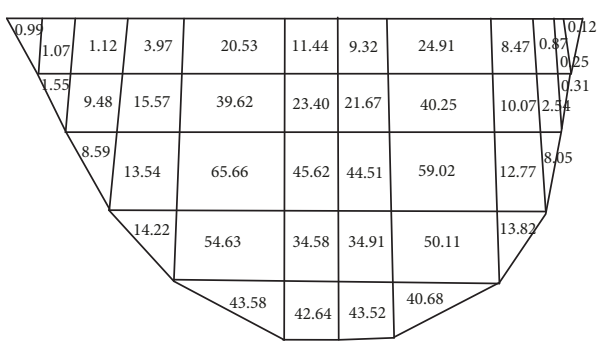

(d) Leader-follower game shape (preferred $f_{3}$ )

FIGURE 1: Comparison of the upstream element strain energy (MJ).

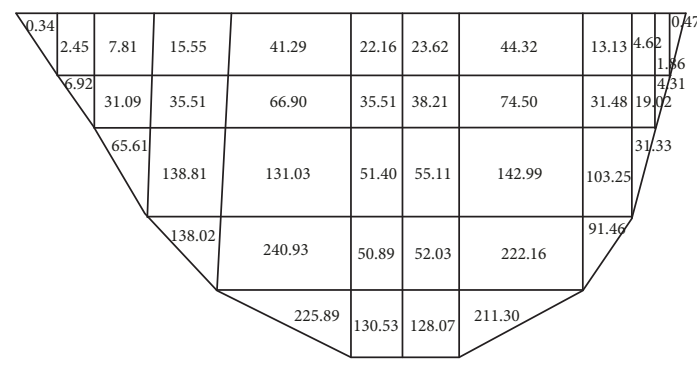

(a) Initial shape

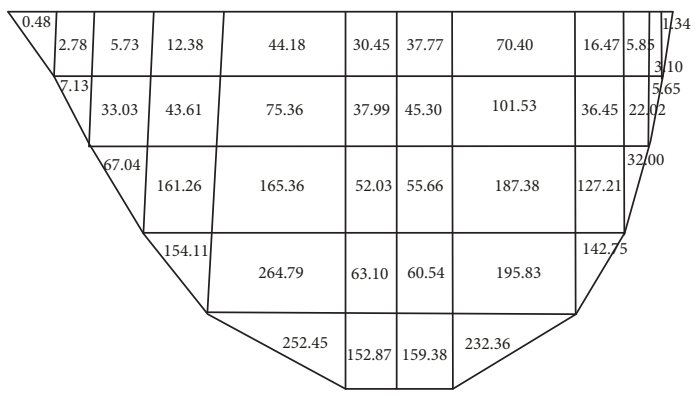

(c) Leader-follower game shape (preferred $f_{2}$ )

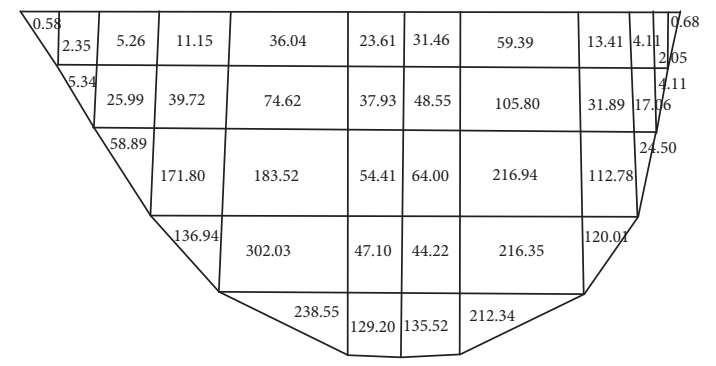

(b) Leader-follower game shape (preferred $f_{1}$ )

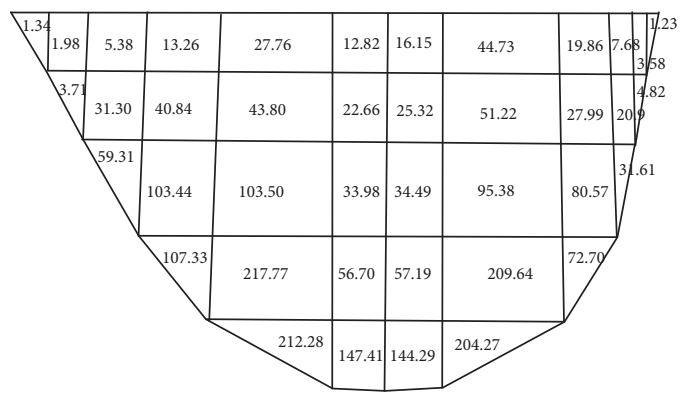

(d) Leader-follower game shape (preferred $f_{3}$ )

Figure 2: Comparison of the downstream element strain energy (MJ). 


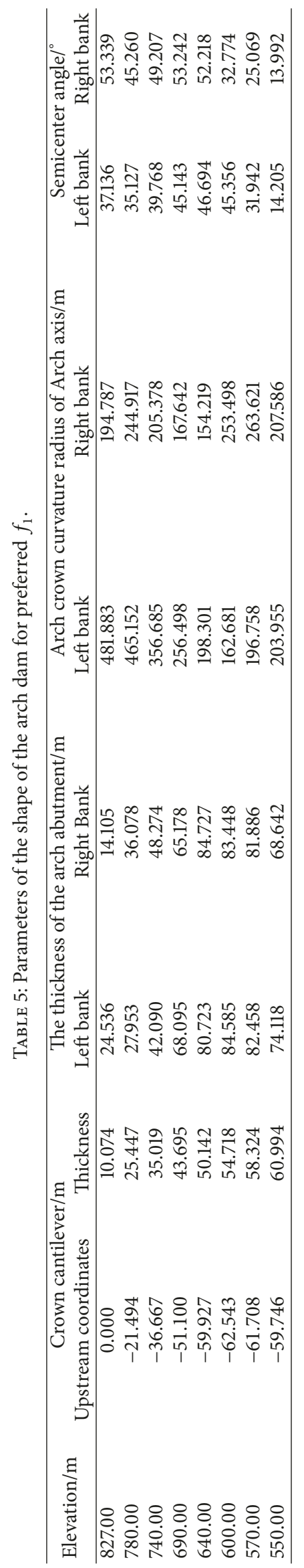




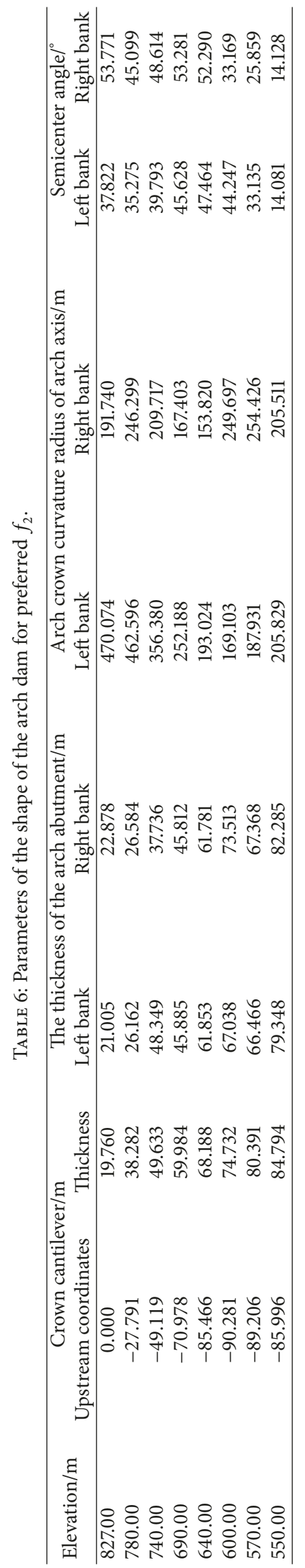




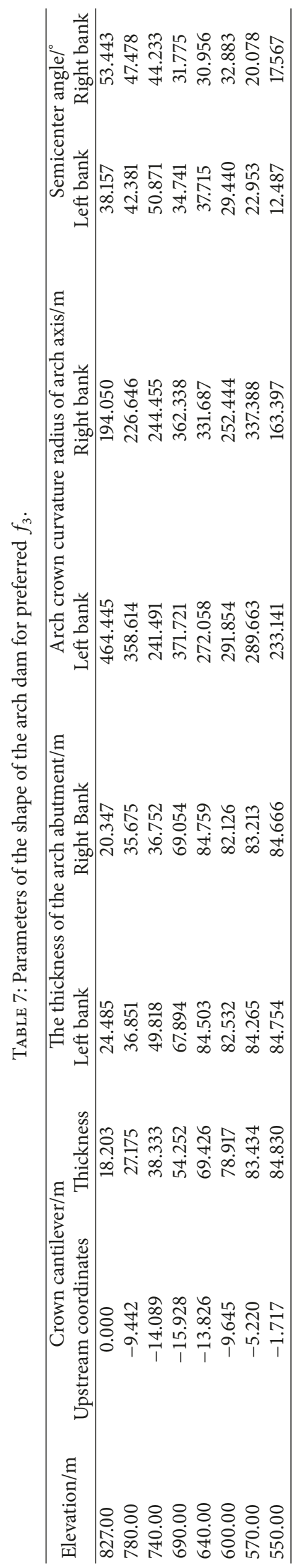




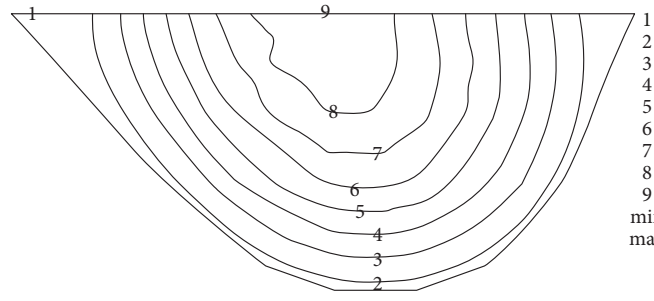

(a) Initial shape

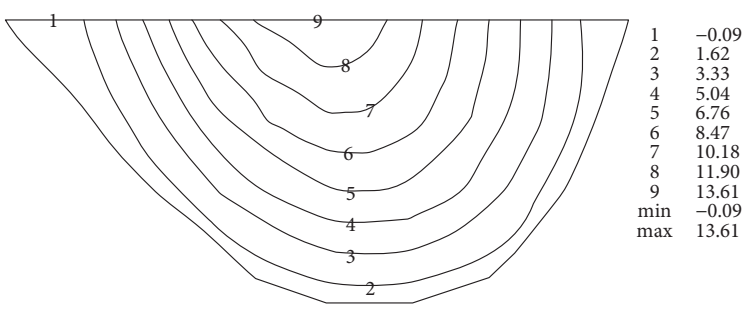

(c) Leader-follower game shape (preferred $f_{2}$ )
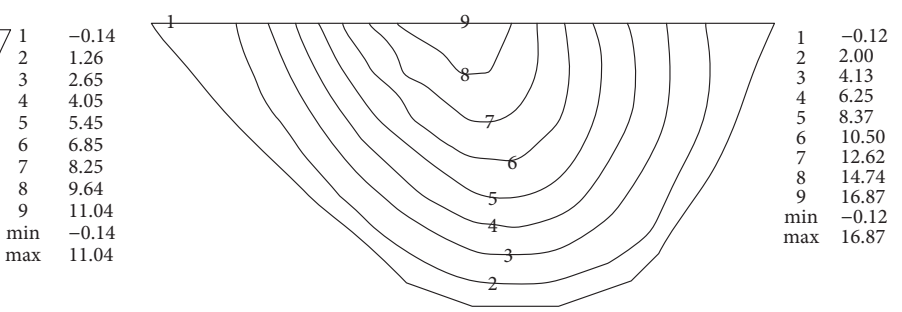

(b) Leader-follower game shape (preferred $f_{1}$ )

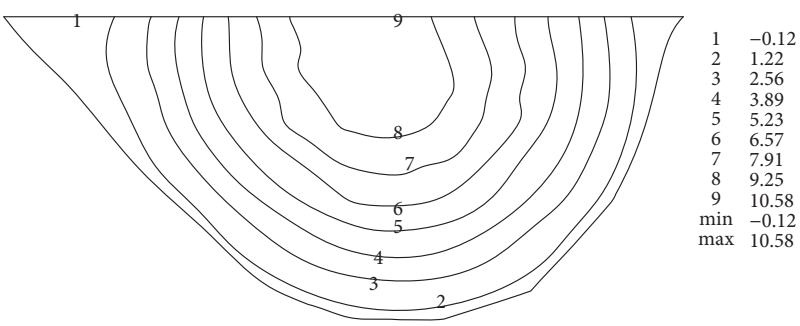

(d) Leader-follower game shape (preferred $f_{3}$ )

Figure 3: Comparison of the displacement along the river of the downstream surface $(\mathrm{cm})$.

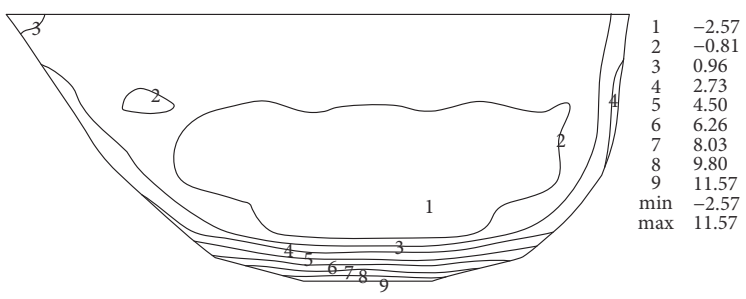

(a) Initial shape

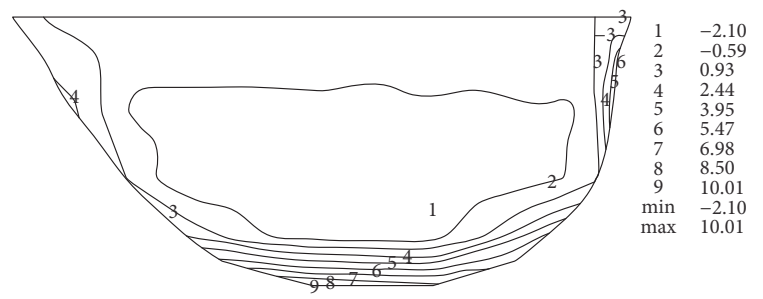

(c) Leader-follower game shape (preferred $f_{2}$ )

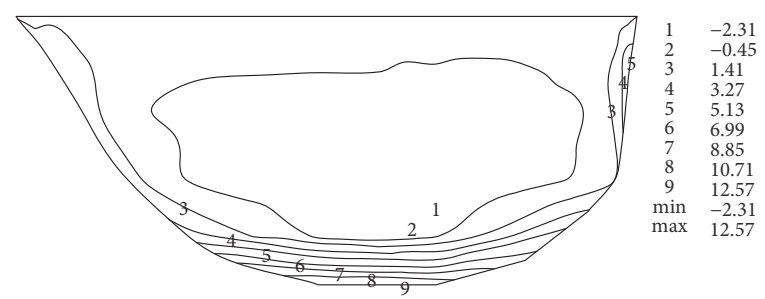

(b) Leader-follower game shape (preferred $f_{1}$ )

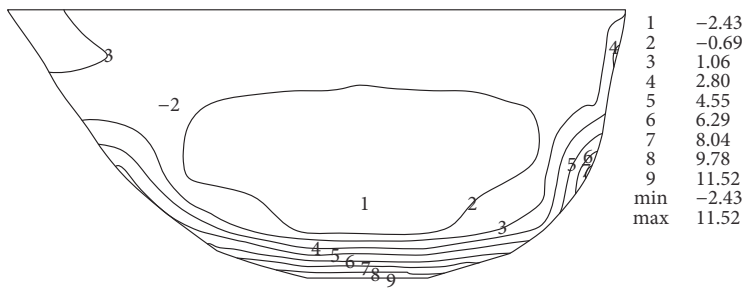

(d) Leader-follower game shape (preferred $f_{3}$ )

FIGURE 4: Comparison of contour line of the upstream surface principal tensile stress (MPa).

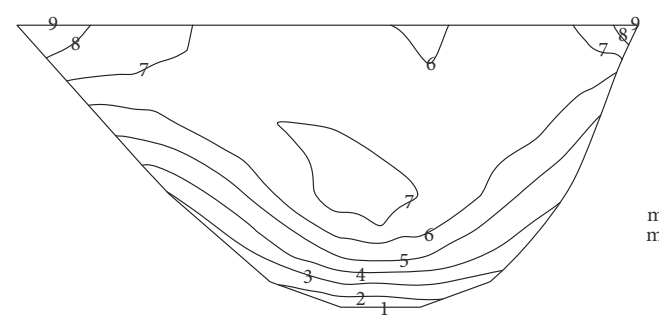

(a) Initial shape

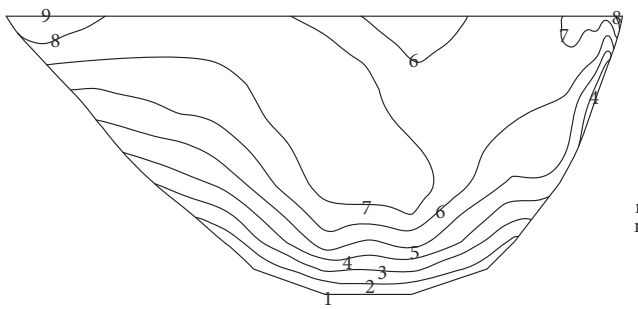

(c) Leader-follower game shape (preferred $f_{2}$ )
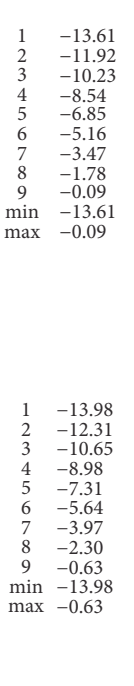

Figure 5: Comparison of the contour line of downstream surface principal compressive stress (MPa).

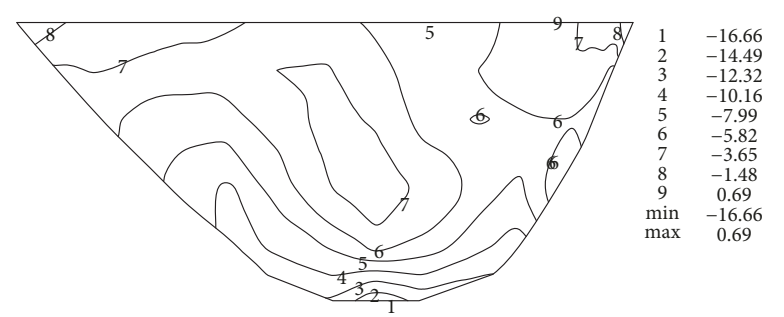

(b) Leader-follower game shape (preferred $f_{1}$ )

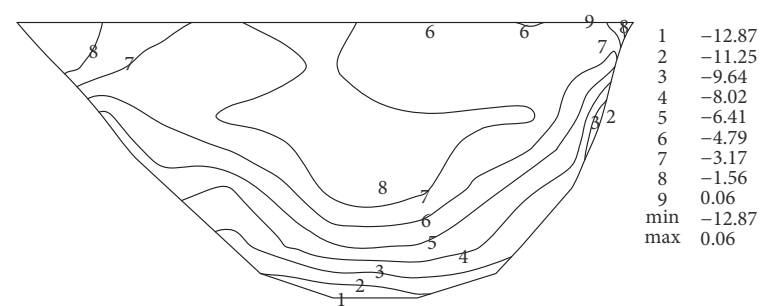

(d) Leader-follower game shape (preferred $f_{3}$ ) 
problem of the follower player is actually a constraint to the optimization problem of the leader player. The strategy set of the leader and the follower forms a nonseparable whole and a relation that restricts each of them.

\section{Conflicts of Interest}

The authors declare no conflicts of interest related to the publication of this paper.

\section{Acknowledgments}

This project was supported by the National Natural Science Foundation of China (Grant no. 61375068 and no. 51605005) and the Talent Project for Higher Education Promotion Program of Anhui Province.

\section{References}

[1] L. Wang, N.-G. Xie, C.-Z. Song, J.-H. Bao, and Y.-W. Cen, "Multi-objective bionics design method of passive suspension parameters based on hybrid behavior game," Structural and Multidisciplinary Optimization, vol. 42, no. 3, pp. 371-386, 2010.

[2] Y. Hu and S. S. Rao, "Game-theory approach for multiobjective optimal design of stationary flat-plate solar collectors," Engineering Optimization, vol. 41, no. 11, pp. 1017-1035, 2009.

[3] A. Clarich, V. Pediroda, L. Padovan, C. Poloni, and J. Periaux, "Application of game strategy in multi-objective robust design optimisation implementing self-adaptive search space decomposition by statistical analysis," in Proceedings of the European Congress on Computational Methods in Applied Sciences and Engineering, ECCOMAS '04, Jyväskylä, July 2004.

[4] C.-S. Lee, "Multi-objective game-theory models for conflict analysis in reservoir watershed management," Chemosphere, vol. 87, no. 6, pp. 608-613, 2012.

[5] X. Li, L. Gao, and W. Li, "Application of game theory based hybrid algorithm for multi-objective integrated process planning and scheduling," Expert Systems with Applications, vol. 39, no. 1, pp. 288-297, 2012.

[6] K. K. Annamdas and S. S. Rao, "Multi-objective optimization of engineering systems using game theory and particle swarm optimization," Engineering Optimization, vol. 41, no. 8, pp. 737752, 2009.

[7] J. Periaux, H. Q. Chen, B. Mantel, M. Sefrioui, and H. T. Sui, "Combining game theory and genetic algorithms with application to DDM-nozzle optimization problems," Finite Elements in Analysis and Design, vol. 37, no. 5, pp. 417-429, 2001.

[8] J. F. Wang, Y. Z. Wu, and J. Périaux, "Distributed evolutionary algorithms for inverse design problems of airfoil in aerodynamics," Acta Aerodynamica Sinica, vol. 21, no. 2, pp. 137-143, 2003.

[9] T. Zhili and D. Jun, "Couplings in Multi-criterion Aerodynamic Optimization Problems Using Adjoint Methods and Game Strategies," Chinese Journal of Aeronautics, vol. 22, no. 1, pp. 1-8, 2009.

[10] Y. Maali, "A multiobjective approach for solving cooperative n-person games," International Journal of Electrical Power \& Energy Systems, vol. 31, no. 10, pp. 608-610, 2009.

[11] L. Chen and S. Li, "A computerized team approach for concurrent product and process design optimization," Computer-Aided Design, vol. 34, no. 1, pp. 57-69, 2002.
[12] N.-G. Xie, R. Meng, Y. Ye, L. Wang, and Y.-W. Cen, "Multiobjective design method based on evolution game and its application for suspension," Structural and Multidisciplinary Optimization, vol. 47, no. 2, pp. 207-220, 2013.

[13] J. F. Wang, Y. Z. Wu, and J. Périaux, "Decentralized multi-point optimization algorithms for multi-airfoil design in aerodynamics," Journal of Astronautics, vol. 24, no. 1, pp. 71-77, 2003.

[14] K. K. Wong, "Bridging game theory and the knapsack problem: a theoretical formulation," Journal of Engineering Mathematics, vol. 91, no. 1, pp. 177-192, 2015.

[15] R. Meng, K. H. Cheong, W. Bao, K. K. L. Wong, L. Wang, and N.-G. Xie, "Multi-objective optimization of an arch dam shape under static loads using an evolutionary game method," Engineering Optimization, vol. 10, pp. 1-17, 2017.

[16] J. Akbari, M. T. Ahmadi, and H. Moharrami, "Advances in concrete arch dams shape optimization," Applied Mathematical Modelling, vol. 35, no. 7, pp. 3316-3333, 2011.

[17] S. Li, L. Ding, L. Zhao, and W. Zhou, "Optimization design of arch dam shape with modified complex method," Advances in Engineering Software, vol. 40, no. 9, pp. 804-808, 2009.

[18] X.-F. Zhang, S.-Y. Li, and Y.-L. Chen, "Optimization of geometric shape of Xiamen arch dam," Advances in Engineering Software, vol. 40, no. 2, pp. 105-109, 2009.

[19] S. M. Seyedpoor, J. Salajegheh, and E. Salajegheh, "Shape optimal design of arch dams including dam-water-foundation rock interaction using a grading strategy and approximation concepts," Applied Mathematical Modelling, vol. 34, no. 5, pp. 1149-1163, 2010.

[20] K. L. Fok and A. K. Chopra, "Earthquake Analysis and Response of Concrete Arch Dams," Tech. Rep. No. UCB/EERC 85/07, Earthquake Engineering Research Center, University of California, Berkeley, 1985.

[21] Z. H. Duron and J. F. Hall, "Experimental and finite element studies of the forced vibration response of morrow point dam," Earthquake Engineering \& Structural Dynamics, vol. 16, no. 7, pp. 1021-1039, 1988. 


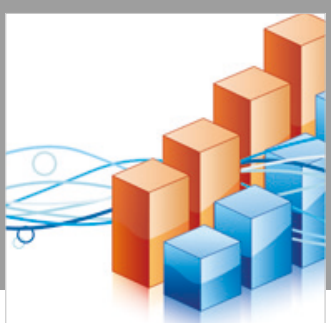

Advances in

Operations Research

\section{-n-m}
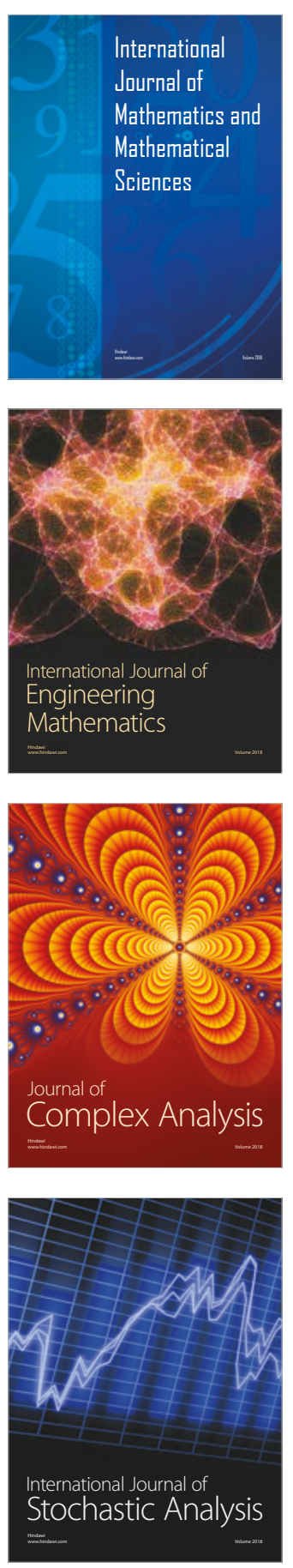
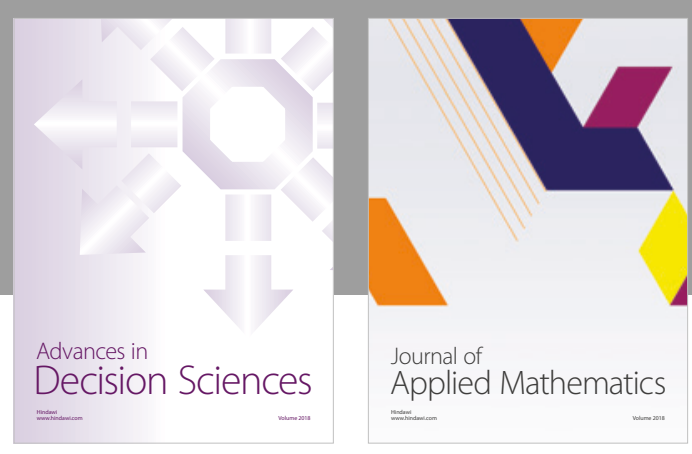

Journal of

Applied Mathematics
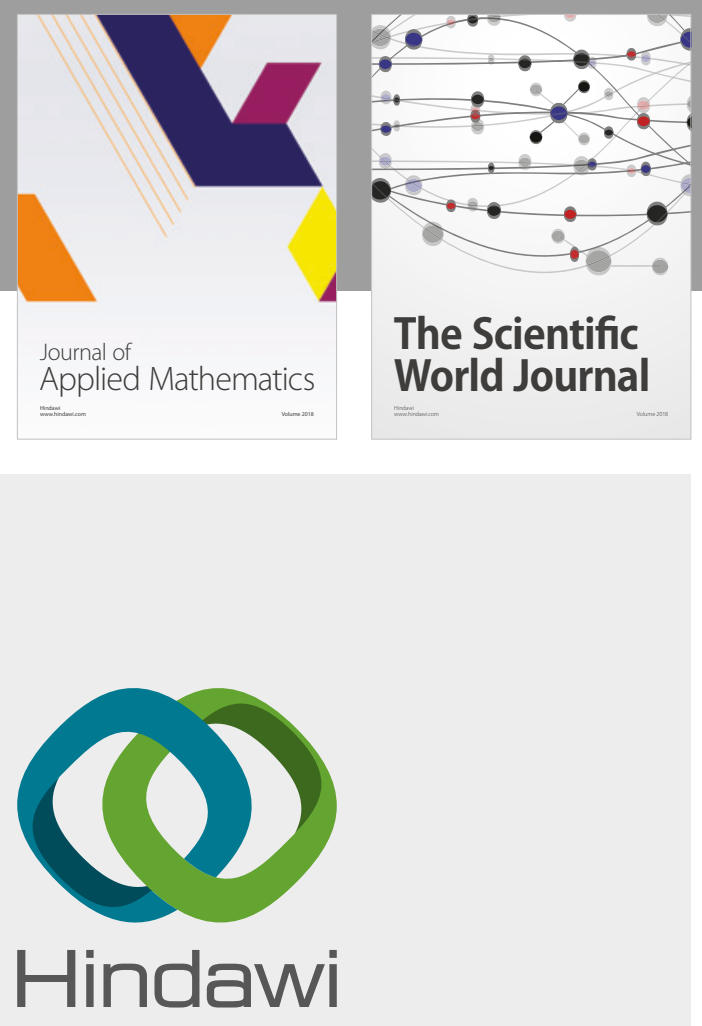

Submit your manuscripts at

www.hindawi.com

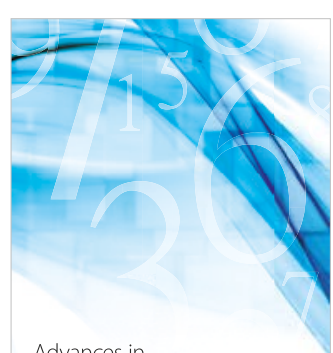

Advances in
Numerical Analysis
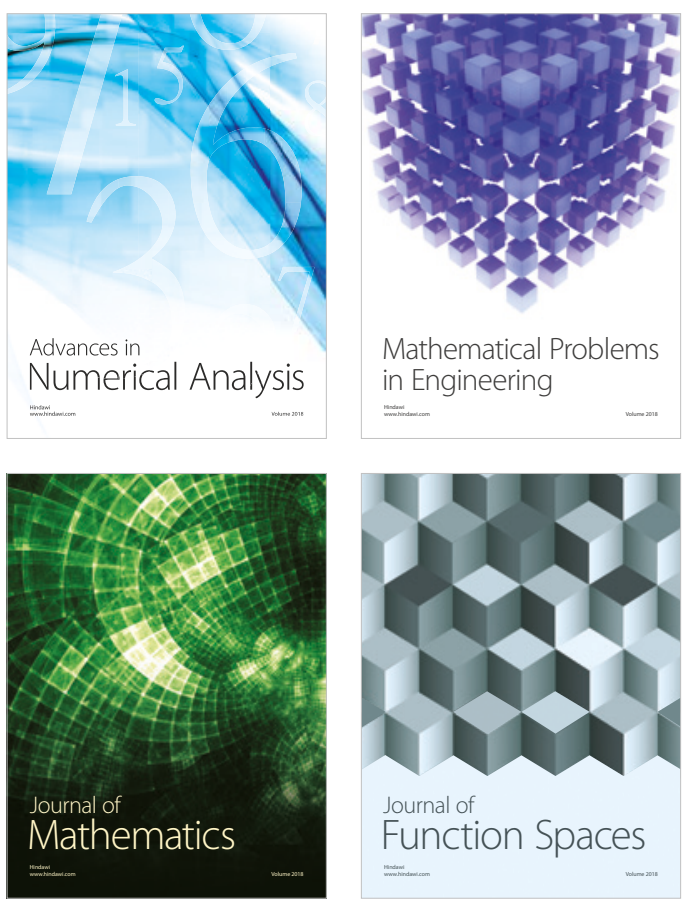

Mathematical Problems in Engineering

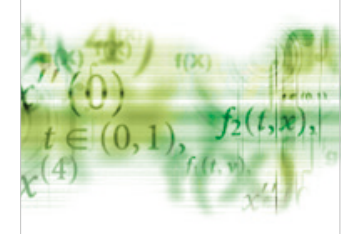

International Journal of

Differential Equations

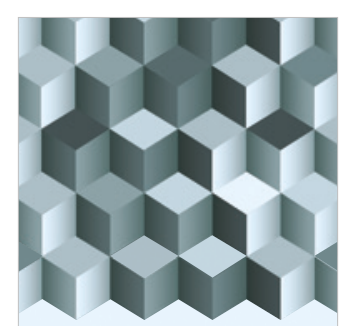

Journal of

Function Spaces

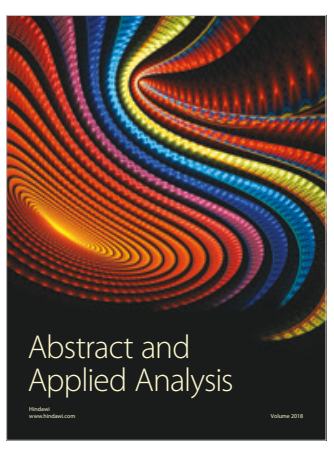

The Scientific

World Journal

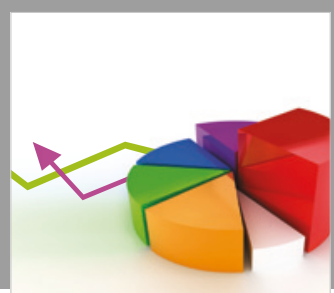

Journal of

Probability and Statistics
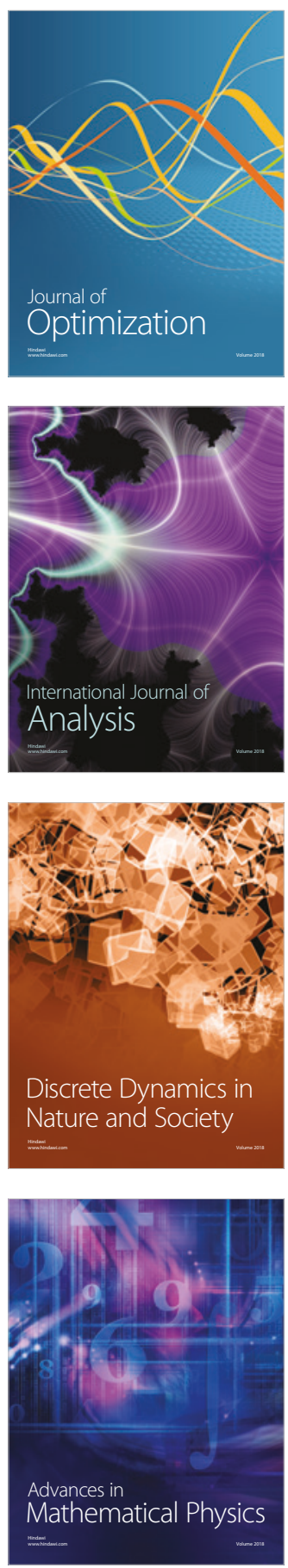